\title{
Diferencia veno-arterial de dióxido de carbono como predictor de gasto cardiaco disminuido en modelo pediátrico experimental
}

\author{
FRANCO DÍAZ ${ }^{1,2}$, ALEJANDRO DONOSO $^{2}$, \\ CRISTÓBAL CARVAJAL ${ }^{3}$, TATIANA SALOMÓN ${ }^{1, a}$, \\ MARÍA TORRES ${ }^{1, a}$, BENJAMÍN ERRANZ ${ }^{3, b}$, PABLO CRUCES ${ }^{2,3}$
}

\section{Veno-arterial difference of carbondioxide as a predictor of low cardiac output in an experimental pediatric model}

Background: Cardiac output (CO) measurement is not a standard of care for critically ill children, but it can be estimated by indirect methods such as venoarterial $p C \mathrm{O}_{2}$ difference $\left(\triangle V A C \mathrm{O}_{2}\right)$. Aim: To determine the correlation between $C O$ and $\triangle V A C O_{2}$ and evaluate the usefulness of $\triangle V A C O_{2}$ in the diagnosis of low $C O$ in an experimental pediatric model. Materials and Methods: Thirty piglets weighing $4.8 \pm 0.35 \mathrm{~kg}$ were anesthetized and monitored with transpulmonary thermodilution. Lung injury was induced with tracheal instillation of Tween $20^{\circledR}$. Serial measurements of central venous and arterial blood gases, as well as $C O$, were obtained at baseline, 1, 2 and $4 \mathrm{~h}$ after lung injury induction. Low cardiac output (LCO) was defined as CO lower than $2.5 \mathrm{~L} / \mathrm{min} / \mathrm{m}^{2}$. Results: There was an inverse correlation between $C O$ and $\triangle V A C O_{2}(r=-0.36, p<0.01) . \triangle V A C O_{2}$ was $14 \pm 8 \mathrm{mmHg}$ in LCO state and $8 \pm 6 \mathrm{mmHg}$ when this condition was not present $(p<0.01)$. Area under the receiver operating characteristic (ROC) curves of $\triangle V A C O_{2}$ and $L C O$ state was 0.78 (0.68-0.86). The best cut-point was $8.9 \mathrm{mmHg}$ to determine LCO with a sensibility 0.78 , specificity 0.7 , positive predictive value 0.27 and negative predictive value 0.96 . Conclusions: In this model there was an inverse correlation between $\triangle V A C \mathrm{O}_{2}$ and $C O$. The best cutoff value to discard LCO was $\triangle V A C O$ of $8.9 \mathrm{mmHg}$, indicating that under this value the presence of LCO is very unlikely.

(Rev Med Chile 2012; 140: 39-44).

Key words: Blood gas monitoring; Carbon Dioxide; Critical Care; Hemodynamics; Cardiac Output.

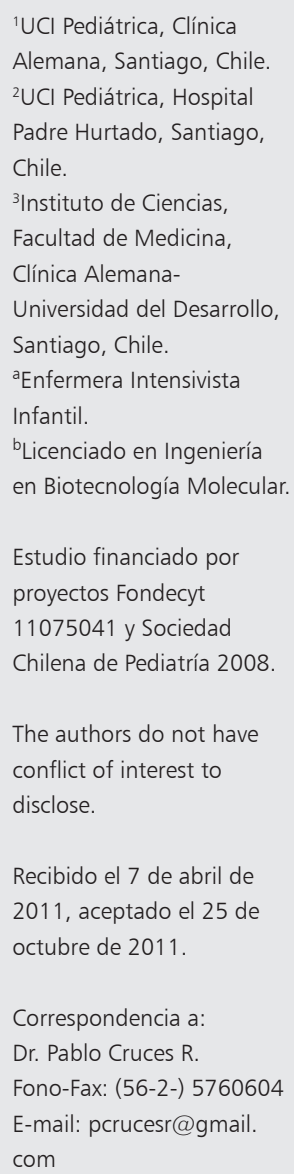

L a determinación del gasto cardiaco (GC) es una herramienta útil para guiar la terapia de pacientes en estado de choque, cuando es incorporado a algoritmos de tratamiento específico ${ }^{1-3}$, permitiendo una aproximación más racional y personalizada a la reanimación con fluidos y catecolaminas ${ }^{4-6}$. Los signos clínicos para reconocer una entrega de oxígeno $\left(\mathrm{DO}_{2}\right)$ inadecuada son inespecíficos y la capacidad para estimar un GC reducido en niños sólo con examen físico es muy limitada ${ }^{7}$. Adicionalmente la medición de GC en pediatría, es poco habitual dado que los métodos son invasivos y pudieran presentar dificultades técnicas (termodilución), poca precisión (principio de Fick directo) o requerir de un alto nivel de entrenamiento (ecocardiografía). Se han propuesto técnicas alternativas para estimar la suficiencia del GC, destacando la diferencia venosa 
central-arterial de $\mathrm{pCO}_{2}\left(\Delta \mathrm{VACO}_{2}\right)$ por su fácil aplicabilidad.

La relación entre GC y $\triangle \mathrm{VACO}_{2}$ deriva del principio de Fick indirecto:

$$
\begin{aligned}
& \mathbf{G C}=\mathbf{V C O}_{2} /\left(\mathbf{C v C O}_{2}-\mathbf{C a C O}_{2}\right) \\
& \mathrm{GC} \alpha 1 / \Delta \mathrm{VACO}_{2}
\end{aligned}
$$

donde $\mathrm{VCO}_{2}$ es la producción de $\mathrm{CO}_{2}, \mathrm{CaCO}_{2}$ y $\mathrm{CvCO}_{2}$ corresponden al contenido arterial y venoso central de $\mathrm{CO}_{2}$, respectivamente. El principio de Fick afirma que el flujo de la difusión de una sustancia es proporcional a la gradiente de la concentración en esa dirección. Aplicando el principio de Fick al GC, éste puede ser calculado por la razón entre la $\mathrm{VCO}_{2}$ y la diferencia entre el contenido arterial y venoso de $\mathrm{CO}_{2}{ }^{8}$. Con una $\mathrm{VCO}_{2}$ constante, una elevación de la $\triangle \mathrm{VACO}_{2}$ representa una condición de falla circulatoria con flujo sistémico reducido, reflejando un barrido tisular de $\mathrm{CO}_{2}$ deficiente.

La $\mathrm{pCO}_{2}$ obtenida de un catéter venoso central, medida en vena cava superior, ha mostrado una buena correlación con su determinación en sangre venosa mixta $^{9-11}$, evitando la instalación de un catéter en arteria pulmonar ${ }^{12-14}$. Existe información insuficiente en pacientes y modelos pediátricos respecto a la capacidad de la $\triangle \mathrm{VACO}_{2}$ para detectar estados de hipodébito.

El objetivo de este estudio es determinar la correlación entre la medición del GC por termodilución transpulmonar y la $\triangle \mathrm{VACO}_{2}$, en un modelo experimental pediátrico de lesión pulmonar aguda y disfunción hemodinámica secundaria.

\section{Métodos}

El protocolo experimental fue aprobado por el Comité de Ética Facultad de Medicina Clínica Alemana-Universidad del Desarrollo y por el Comité Asesor de Bioética de CONICYT. Los animales recibieron cuidados en conformidad con los "Guiding Principles in the Care and Use of Laboratory Animals" adoptados por la American Physiological Society.

\section{Sujetos}

Treinta cerdos Large-White, menores de un mes de vida, de 4,8 $\pm 0,35 \mathrm{~kg}$.

\section{Anestesia}

Después de premedicación con ketamina (20 $\mathrm{mg} / \mathrm{kg})$ y acepromazina $(1,1 \mathrm{mg} / \mathrm{kg})$ intramuscular, anestesia y relajación fueron mantenidas con infusión continua de propofol $(10 \mathrm{mg} / \mathrm{kg} / \mathrm{h})$, fentanilo $(4 \mu \mathrm{g} / \mathrm{kg} / \mathrm{h})$ y pancuronium $(0,2 \mathrm{mg} /$ $\mathrm{kg} / \mathrm{h}$ ). Hidratación fue mantenida con solución salina normal a $10 \mathrm{~mL} / \mathrm{kg} / \mathrm{h}$. Temperatura se mantuvo a $37,0 \pm 0,7^{\circ} \mathrm{C}$ usando métodos convectivos convencionales.

\section{Preparación quirúrgica}

Se infiltró en forma tópica lidocaína 1\% (1 mL/ $\mathrm{kg}$ ) y se realizó traqueostomía. La vena yugular izquierda fue denudada y se instaló un catéter $(4 \mathrm{~F})$ (Arrow, Reading, PA, USA), procurando ubicación extra cardiaca en control radiológico. Con técnica similar se instaló catéter arterial $(4 \mathrm{~F})$ con punta termistor en extremo distal ( PiCCO $^{\circledR}$ PV2014L08, Pulsion Medical Systems, Munich, Germany) en arteria axilar derecha y se conectó a monitor $\mathrm{PiCCO}^{\circ}$ plus para termodilución transpulmonar (TDTP).

\section{Mediciones}

Los signos vitales fueron monitorizados empleando el monitor Infinity Delta XL (Dräger Medical, Germany), registrando la Frecuencia Cardiaca (FC), Presión Arterial Media (PAM) y Presión Venosa Central (PVC).

\section{Medición de gasto cardiaco}

Se realizó TDTP con $5 \mathrm{ml}$ de solución salina $0,9 \%$ fría $\left(<8^{\circ} \mathrm{C}\right)$ por catéter venoso yugular, administradas en forma aleatoria en el ciclo respiratorio. Las mediciones de GC fueron consideradas válidas cuando las tres medidas aisladas difirieron menos de $10 \%$ del promedio. En caso de una diferencia $>10 \%$, se realizó una medición adicional. Se consideraron valores indexados según superficie corporal. Área de superficie corporal fue calculada usando la siguiente fórmula: área de superficie corporal $=\mathrm{K} /$ peso $(\text { en kilogramos) })^{2 / 3}$, donde $\mathrm{K}=$ 0,112 para $\operatorname{cerdos}^{15}$. El estado de hipodébito fue definido como un GC menor de $2,5 \mathrm{~L} / \mathrm{min} / \mathrm{m}^{2}$.

\section{Inducción de la lesión pulmonar aguda}

Luego de pre-oxigenación con $\mathrm{FIO}_{2}$ 100\%, se realizó inducción de la lesión pulmonar aguda con instilación traqueal de $2 \mathrm{~mL} / \mathrm{kg}$ Tween $20^{\circledR}$ al $10 \%$ en solución fisiológica, en dos alícuotas iguales en 
decúbito lateral izquierdo y derecho, para obtener un índice de $\mathrm{PaO}_{2} / \mathrm{FIO}_{2}$ entre $100-200 \mathrm{mmHg}$ con PEEP $10 \mathrm{cmH}_{2} \mathrm{O}$.

\section{Protocolo de ventilación}

Se empleó ventilador mecánico EVITA XL (Dräger Medical, Germany) en modo controlado por volumen y regulado por presión (Autoflow), $\mathrm{FIO}_{2} 100 \%$, volumen corriente $\left(\mathrm{V}_{\mathrm{T}}\right) 6 \mathrm{~mL} / \mathrm{kg}$, PEEP de $10 \mathrm{cmH}_{2} \mathrm{O}$, frecuencia respiratoria (FR) 30 por minuto y tiempo inspiratorio (TI) 0,75 segundos.

\section{Mediciones de gasometría y mecánica pulmonar}

Se midió gas arterial y venoso central empleando i-STAT ${ }^{\circledR}$ Cartridges EG6 (Abbott Laboratories) al inicio y luego 1,2 y $4 \mathrm{~h}$ post inducción de la lesión pulmonar aguda. Simultáneamente se midió el GC por TDTP. La compliance estática del sistema respiratorio (Cst) fue medida como la razón entre $V_{T}$ espirado y la diferencia entre presiones inspiratoria y espiratoria final de las vías aéreas, medidas tras una pausa inspiratoria y espiratoria de 4 segundos respectivamente. Luego, aún bajo anestesia (Estado 3 - Plano 2), los animales fueron sacrificados por perfusión de cloruro de potasio al 10\% hasta la detección de la fibrilación ventricular o asistolia.

\section{Análisis estadístico}

Normalidad fue evaluada usando test de Anderson Darling. Se realizó correlación de Pearson entre GC y $\triangle \mathrm{VACO}_{2}$. El test de Kruskal-Wallis se usó para análisis de subgrupos. La capacidad de la $\triangle \mathrm{VACO}_{2}$ para discriminación de hipodébito se analizó mediante el cálculo del área bajo la curva receiver operating characteristic (AUC-ROC). El análisis estadístico se realizó con programa STATA $10^{\oplus}$ (StataCorp LP, Texas, USA). Los valores fueron expresados como media \pm SEM. Se consideró significativo un $\mathrm{p}<0,05$.

\section{Resultados}

Todos los animales completaron el protocolo experimental. Posterior a la lesión pulmonar aguda hubo una reducción significativa de la $\mathrm{PaO}_{2} / \mathrm{FiO}_{2}$ $(345 \pm 61$ contra $155 \pm 37 \mathrm{mmHg}, \mathrm{p}<0,01)$ y de la compliance pulmonar $(1,6 \pm 0,6$ contra $0,9 \pm$ $0,2 \mathrm{~mL} / \mathrm{cmH}_{2} \mathrm{O} / \mathrm{kg}, \mathrm{p}<0,01$ ), asociado a un incremento en la $\mathrm{PaCO}_{2}(50 \pm 10$ contra $66 \pm 12 \mathrm{mmHg}$, $\mathrm{p}<0,01)$ con acidosis respiratoria concomitante ( $\mathrm{pH}$ de sangre arterial 7,29 $\pm 0,08$ contra 7,18 \pm $0,1, \mathrm{p}<0,01)$.

Se realizaron un total de 120 mediciones hemodinámicas. El GC fue $3,74 \pm 1,12 \mathrm{~L} / \mathrm{min} / \mathrm{m}^{2}$ (rango $1,62-7,44)$ y la $\Delta \mathrm{VACO}_{2}$ fue $7,3 \pm 5,4 \mathrm{mmHg}$ (rango 0-27). Hubo una correlación inversa entre el GC y la $\triangle \mathrm{VACO}_{2}(\mathrm{r}=-0,36 ; \mathrm{p}=0,009)$ (Figura 1 ). En condiciones de hipodébito, la $\triangle \mathrm{VACO}_{2}$ fue $14 \pm 8 \mathrm{mmHg}$, mientras que en ausencia de esta condición fue $8 \pm 6 \mathrm{mmHg}(\mathrm{p}=0,0058)$.

La curva $R O C$ calculada para $\triangle \mathrm{VACO}$, e hipodébito tuvo un área bajo la curva de 0,78 (95\%IC: 0,68-0,86) (Figura 2). El mejor punto de corte de

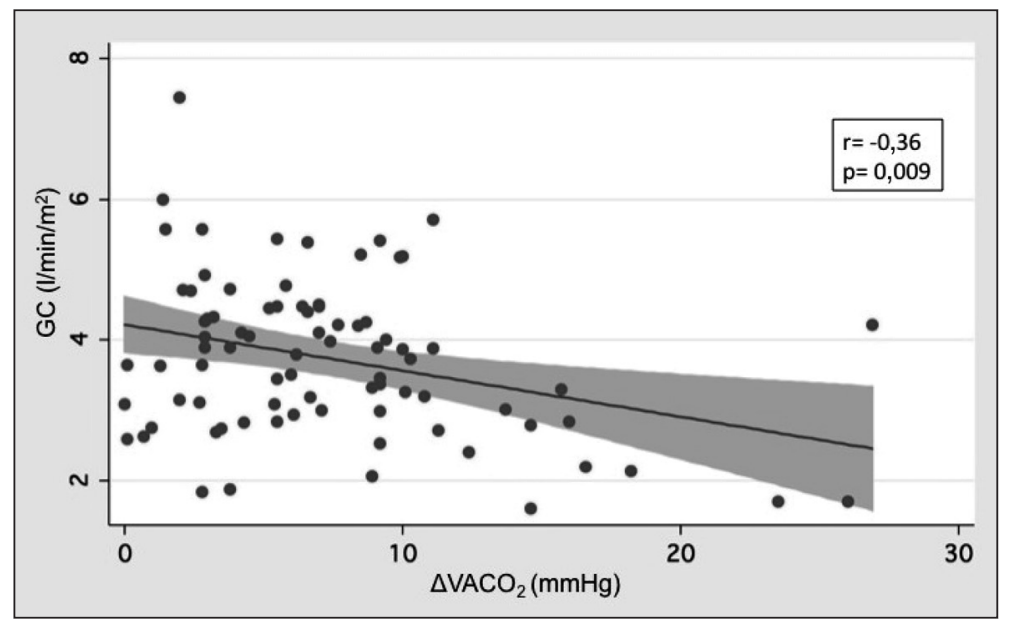

Figura 1. Correlación entre la diferencia veno-arterial de $\mathrm{CO}_{2}$ y gasto cardiaco en modelo experimental. $\triangle \mathrm{VACO}_{2}$ : diferencia veno-arterial de $\mathrm{CO}_{2} ; \mathrm{GC}$ : Gasto cardiaco. Línea central representa correlación y zona sombreada rango intercuartil. 


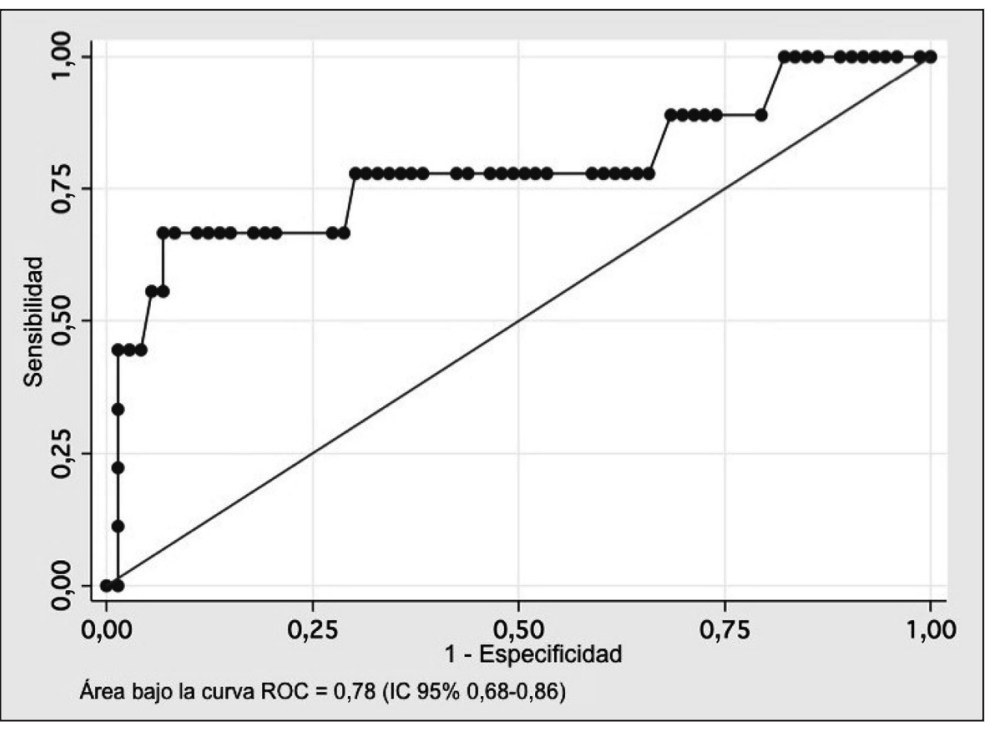

Figura 2. Curva ROC para la diferencia veno-arterial de $\mathrm{CO}_{2}$ e hipodébito en modelo experimental. $\triangle \mathrm{VACO}_{2}$ seleccionado fue $8,9 \mathrm{mmHg}$, con una sensibilidad de 0,78 , una especificidad de 0,7 , un valor predictivo positivo de $0,27 \mathrm{y}$ un valor predictivo negativo de 0,96 .

\section{Discusión}

En este modelo experimental pediátrico de lesión pulmonar aguda y disfunción hemodinámica, los principales hallazgos fueron: 1) Existe una correlación significativa entre la $\triangle \mathrm{VACO}_{2} \mathrm{y}$ el GC ante variadas condiciones de flujo circulatorio (GC entre $1,62-7,44 \mathrm{~L} / \mathrm{min} / \mathrm{m}^{2}$ ) y 2 ) una $\triangle \mathrm{VACO}_{2}$ menor a $8,9 \mathrm{mmHg}$ logra descartar un GC menor que $2,5 \mathrm{~L} / \mathrm{min} / \mathrm{m}^{2}$. El valor predictivo negativo de 0,96 refleja que bajo este punto de corte se descarta con muy buena fiabilidad un estado de hipodébito.

Se ha descrito una correlación inversa entre la $\triangle V_{A C O}$ y el GC en pacientes adultos que padecen una sepsis ${ }^{11}$ o durante su recuperación de cardiocirugía $^{12}$. En nuestro estudio la correlación entre estas determinaciones fue muy similar a la descrita en pacientes cardioquirúrgicos ${ }^{12}$ y bastante menor a la descrita en pacientes sépticos ${ }^{11}$. El punto de corte identificado para detectar hipodébito fue mayor que lo previamente publicado, entre 6 y 7 $\mathrm{mmHg} \mathrm{g}^{11,13,16}$. Estas diferencias pudieran atribuirse a que estudiamos un modelo pediátrico de disfun- ción pulmonar primaria, mientras que los otros estudios incluyeron predominantemente pacientes adultos con shock séptico o cardiogénico. En adultos una $\triangle \mathrm{VACO}$, elevada se asocia a persistencia del metabolismo anaeróbico en pacientes con shock séptico $^{14} \mathrm{y}$ a complicaciones postoperatorias en pacientes quirúrgicos de alto riesgo, aún con metas de reanimación cumplidas ${ }^{13,15}$. Sin embargo, estos hallazgos no pueden extrapolarse a niños gravemente enfermos dadas las marcadas diferencias que existen entre la respuesta hemodinámica de pacientes pediátricos y adultos.

La correlación entre $\triangle \mathrm{VACO}_{2}$ y GC fue sólo moderada lo que es comprensible dado que la $\mathrm{VCO}_{2}$ no es un parámetro controlado, tanto en condiciones clínicas como experimentales de disfunción respiratoria y hemodinámica. A pesar de ello, la $\triangle \mathrm{VACO}$ nos permite una estimación de la suficiencia del GC, aportando información útil y rápida, mientras se realiza un procedimiento más definitorio, en caso de ser necesario. La capacidad de extracción de $\mathrm{O}_{2}$ por los tejidos está determinada por el balance entre el flujo sanguíneo microvascular y la demanda de oxígeno regional, adaptándose el GC a ésta última ${ }^{17}$. Cuando la $\mathrm{DO}_{2}$ falla en un nivel crítico, el consumo de oxígeno $\left(\mathrm{VO}_{2}\right)$ empieza a disminuir, desarrollándose anaerobiosis. Las medidas terapéuticas destinadas a revertir el metabolismo anaeróbico han demostrado reducir la duración de la hospitalización, 
la disfunción de órganos ${ }^{18}$ y la mortalidad ${ }^{19}$. Por ello muchos clínicos optan por una aproximación titulada individualmente y basada en el análisis integrado de pruebas complementarias globales y regionales para una completa comprensión de su fisiopatología hemodinámica. Han transcurrido casi dos décadas desde que se describió que el shock se asociaba a un incremento de la $\mathrm{pCO}_{2}$ tisular y venosa ${ }^{20}$. Se postula que la hipercapnia venosa ocurriría al ser tamponados los hidrogeniones producidos por el metabolismo anaerobio, para mantener el equilibrio ácido-base en células con un potencial redox disminuido. Así como la $\mathrm{pCO}_{2}$ arterial depende de la ventilación alveolar y del intercambio gaseoso pulmonar, la $\mathrm{pCO}_{2}$ venosa depende del flujo circulatorio y no necesariamente de la hipoxia hipoxémica, correlacionándose inversamente al GC en falla circulatoria ${ }^{14,21-30}$, hallazgo reafirmado en este modelo pediátrico. Concordantemente se ha propuesto un rol de la $\Delta \mathrm{VACO}_{2}$ en guiar la terapia de reanimación, de manera complementaria a la saturación venosa central de oxígeno ${ }^{13}$.

La principales limitaciones de este estudio fueron propias de un modelo primario de falla respiratoria con una disfunción hemodinámica heterogénea, semejante a lo que podemos apreciar en la práctica clínica. Creemos importante enfatizar: 1) La acidosis respiratoria, propia de un modelo de lesión pulmonar aguda, fue un hallazgo frecuente, pero no interfirió mayormente con la confiabilidad de las determinaciones, pudiendo incrementar levemente el punto de corte; 2) El empleo de TDTP no es el estándar de oro para medir GC, sin embargo, este método está validado en pacientes y modelos pediátricos ${ }^{31-33}$;3) El alto valor predictivo negativo de la $\triangle \mathrm{VACO}_{2}$ pudo estar influenciado por una baja incidencia relativa de GC menor que $2,5 \mathrm{~L} / \mathrm{min} / \mathrm{m}^{2}$. Es importante destacar que esta incidencia fue similar a la reportada por otros autores ${ }^{11}$; 4) Aunque exista una correlación significativa entre $\triangle \mathrm{VACO}{ }_{2} \mathrm{y}$ GC, ésta fue sólo moderada, lo que es compatible con la fisiopatología del modelo desarrollado; 5) Finalmente, debemos agregar las limitaciones propias de extrapolar la información desde modelos experimentales a pacientes gravemente enfermos.

A pesar de esto nos parece de gran importancia incorporar esta determinación en la detección de un estado de hipodébito con un método poco invasivo, simple y de bajo costo, como la $\triangle \mathrm{VACO}_{2}$.
Futuros estudios debieran dilucidar si la inclusión de la $\triangle \mathrm{VACO}_{2}$ en algoritmos de tratamiento y resucitación guiada por objetivos pudiera complementar la información aportada por la saturación venosa central de oxígeno y mejorar el pronóstico de niños gravemente enfermos.

\section{Conclusión}

En este modelo experimental, la $\triangle \mathrm{VACO}_{2}$ obtenida de la circulación venosa central se correlacionó en forma inversa con el GC, siendo 8,9 $\mathrm{mmHg}$ el mejor punto de corte para descartar un estado de bajo GC, bajo este valor la presencia de hipodébito es muy improbable. En ausencia de monitorización invasiva de GC, esta medición pudiera emplearse para su estimación, más aún si consideramos su fácil acceso en cuidados intensivos.

\section{Referencias}

1. Carcillo JA, Fields AI; American College of Critical Care Medicine Task Force Committee Members. Clinical practice parameters for hemodynamic support of pediatric and neonatal patients in septic shock. Crit Care Med 2002; 30: 1365-78.

2. de Oliveira CF, de Oliveira DS, Gottschald AF, Moura JD, Costa GA, Ventura AC, et al. ACCM/PALS haemodynamic support guidelines for paediatric septic shock: an outcomes comparison with and without monitoring central venous oxygen saturation. Intensive Care Med 2008; 34: 1065-75.

3. Nguyen HB, Rivers EP, Abrahamian FM, Moran GJ, Abraham E, Trzeciak S, et al. Emergency Department Sepsis Education Program and Strategies to Improve Survival (ED-SEPSIS) Working Group: Severe sepsis and septic shock: review of the literature and emergency department management guidelines. Ann Emerg Med 2006; 48: 28-54.

4. Thompson AE. Pulmonary artery catheterization in children. New Horiz 1997; 5: 244-50.

5. Tibby SM, Murdoch IA. Monitoring cardiac function in intensive care. Arch Dis Child 2003; 88: 46-52.

6. Ceneviva G, Paschall JA, Maffei F, Carcillo JA. Hemodynamic support in fluid refractory pediatric septic shock. Pediatrics 1998; 102: e19.

7. Tibby SM, Hatherill M, Marsh MJ, Murdoch IA. Clinicians' abilities to estimate cardiac index in ventilated children and infants. Arch Dis Child 1997; 77: 516-8. 
8. Teboul JL, Monnet X. Clinical use of venoarterial $\mathrm{pCO}_{2}$ difference in septic shock. Yearbook of intensive care and emergency medicine 2003, 574-82.

9. Reinhart K, Rudolph T, Bredle DL, Hannemann L, Cain SM. Comparison of central-venous to mixed-venous oxygen saturation during changes in oxygen supply/ demand. Chest 1989; 95: 1216-21.

10. Ho KM, Harding R, Chamberlain J. A comparison of central venous-arterial and mixed venous-arterial carbon dioxide tension gradient in circulatory failure. Anaesth Intensive Care 2007; 35: 695-701.

11. Cuschieri J, Rivers E, Donnino M, Katilius M, Jacobsen G, Nguyen HB, et al. Central venous-arterial carbon dioxide difference as an indicator of cardiac index. Intensive Care Med 2005; 31: 818-22.

12. Yazigi A, Abou-Zeid H, Haddad F, Madi-Jebara S, Hayeck G, Jabbour K. Correlation between central venous-arterial carbon dioxide tension gradient and cardiac index changes following fluid therapy. Ann Card Anaesth 2010; 13: 269-71.

13. Futier E, Robin E, Jabaudon M, Guerin R, Petit A, Bazin JE, et al. Central venous $\mathrm{O}_{2}$ saturation and venousto-arterial $\mathrm{CO}_{2}$ difference as complementary tools for goal-directed therapy during high-risk surgery. Crit Care 2010; 14: R193.

14. Vallée F, Vallet B, Mathe O, Parraguette J, Mari A, Silva $\mathrm{S}$, et al. Central venous-to-arterial carbon dioxide difference: an additional target for goal-directed therapy in septic shock? Intensive Care Med 2008; 34: 2218-25.

15. Roch A, Michelet P, Lambert D, Delliaux S, Saby C, Perrin $\mathrm{G}$, et al. Accuracy of the double indicator method for measurement of extravascular lung water depends on the type of acute lung injury. Crit Care Med 2004; 32: 811-7.

16. Mekontso-Dessap A, Castelain V, Anguel N, Bahloul M, Schauvliege F, Richard C, et al. Combination of venoarterial $\mathrm{PCO}_{2}$ difference with arteriovenous $\mathrm{O}_{2}$ content difference to detect anaerobic metabolism in patients. Intensive Care Med 2002; 28: 272-7.

17. Humer MF, Phang PT, Friesen BP, Allard MF, Goddard CM, Walley KR. Heterogeneity of gut capillary transit times and impaired gut oxygen extraction in endotoxemic pigs. J Appl Physiol 1996; 81: 895-904.

18. Pölönen P, Ruokonen E, Hippeläinen M, Pöyhönen M, Takala J. A Prospective, Randomized Study of GoalOriented Hemodynamic Therapy in Cardiac Surgical Patients. Anesth Analg 2000; 90: 1052-9.

19. Rivers E, Nguyen B, Havstad S, Ressler J, Muzzin A, Knoblich B, et al. Early goal-directed therapy in the treatment of severe sepsis and septic shock. N Engl J Med 2001; 345: 1368-77.
20. Johnson BA, Weil MH. Redefining ischemia due to circulatory failure as dual defects of oxygen deficits and of carbon dioxide excesses. Crit Care Med 1991; 19: 1432-8.

21. Durkin R, Gergits MA, Reed JF 3rd, Fitzgibbons J. The relationship between the arteriovenous carbon dioxide gradient and cardiac index. J Crit Care 1993; 8: 217-21.

22. Bakker J, Vincent JL, Gris P, León M, Coffernils M, Kahn RJ. Veno-arterial carbon dioxide gradient in human septic shock. Chest 1992; 101: 509-15.

23. Cavaliere F, Martinelli L, Guarneri S, Varano C, Rossi $\mathrm{M}$, Schiavello R. Arterial-venous $\mathrm{PCO}_{2}$ gradient in early postoperative hours following myocardial revascularization. J Cardiovasc Surg (Torino) 1996; 37: 499-503.

24. Ducey JP, Lamiell JM, Gueller GE. Arterial-venous carbon dioxide tension difference during severe hemorrhage and resuscitation. Crit Care Med 1992; 20: 518-22.

25. Halmagyi DF, Kennedy M, Varga D. Hidden hypercapnia in hemorrhagic hypotension. Anesthesiology 1970; 33: 594-601.

26. Jacob SV, Hornby L, Lands LC. Estimation of mixed venous $\mathrm{PCO}_{2}$ for determination of cardiac output in children. Chest 1997; 111: 474-80.

27. Sato S, Okubo N, Satsumae T, Kumagai M, Yamamoto $\mathrm{S}$, Nakayama $\mathrm{H}$, et al. Arteriovenous differences in $\mathrm{PCO}_{2}$ and cardiac output during CPR in the dog. Resuscitation 1994; 27: 255-9.

28. Sato Y, Weil MH, Tang W. Tissue hypercarbic acidosis as a marker of acute circulatory failure (shock). Chest 1998; 114: 263-74.

29. Weil MH, Rackow EC, Trevino R, Grundler W, Falk JL, Griffel MI. Difference in acid-base state between venous and arterial blood during cardiopulmonary resuscitation. N Engl J Med 1986; 315: 153-6.

30. Rackow EC, Astiz ME, Mecher CE, Weil MH. Increased venous-arterial carbon dioxide tension difference during severe sepsis in rats. Crit Care Med 1994; 22: 121-5.

31. Tibby SM, Hatherill M, Marsh MJ, Morrison G, Anderson D, Murdoch IA. Clinical validation of cardiac output measurements using femoral artery thermodilution with direct Fick in ventilated children and infants. Intensive Care Med. 1997; 23: 987-91.

32. Rupérez M, López-Herce J, García C, Sánchez C, García E, Vigil D. Comparison between cardiac output measured by the pulmonary arterial thermodilution technique and that measured by the femoral arterial thermodilution technique in a pediatric animal model. Pediatr Cardiol. 2004; 25: 119-23.

33. Lemson J, de Boode WP, Hopman JC, Singh SK, van der Hoeven JG. Validation of transpulmonary thermodilution cardiac output measurement in a pediatric animal model. Pediatr Crit Care Med 2008; 9: 313-9. 